\title{
Biosurveillance Ecosystem (BSVE) Workflow Analysis
}

\author{
Timothy Dasey*1, Hayley Davison Reynolds ${ }^{1}$, Nancy Nurthen², Christopher Kiley ${ }^{2}$ and John \\ Silva ${ }^{3}$
}

${ }^{1}$ Chemical and Biological Defense Systems, MIT Lincoln Laboratory, Lexington, MA, USA; ${ }^{2}$ DTRA Chemical \& Biological

Technologies Directorate, Ft. Belvoir, VA, USA; ${ }^{3}$ Silva Consulting Services, LLC, Sykesville, MD, USA

\section{Introduction}

The Defense Threat Reduction Agency Chemical and Biological Technologies Directorate (DTRA CB) has initiated the Biosurveillance Ecosystem (BSVE) research and development program. Operational biosurveillance capability gaps were analyzed and the required characteristics of new technology were outlined, the results of which will be described in this contribution.

\section{Methods}

Work process flow diagrams, with associated explanations and historical examples, were developed based on in-person, structured interviews with public health and preventative medicine analysts from a variety of Department of Defense (DoD) organizations, and with one organization in the Department of Health and Human Services (DHHS) and with a major U.S. city health department. The particular nuanced job characteristics of each organization were documented and subsequently validated with the individual analysts. Additionally, the commonalities across different organizations were described in meta-workflow diagrams and descriptions.

\section{Results}

Two meta-workflows were evident from the analysis. In the first type, epidemiologists identify and characterize health-impacting events, determine their cause, and determine community-level responses to the event. Analysts of this type monitor information (primarily statistical case information) from syndromic or disease reporting system or other sources to determine whether there are any unusual diseases or clusters of disease outbreaks in the jurisdiction. This workflow involved three consecutive processes: triage, analysis and reporting. Investigation and response processes to disease outbreaks are both parallel and overlapping in many circumstances. In the second meta-workflow type, analysts monitor for a potential health event through text-based sources and data reports within their particular area of responsibility. This surveillance activity is often interspersed with other activities required of their job. They may generate a daily/weekly/monthly report or only report when an event is detected that requires notification/response. There are similar triage, analysis and reporting workflow stages to the first meta-workflow type, but in contrast these analysts are focused on informing leadership and response in the form of policy modification. They are also subject to answering leadership-driven biosurveillance queries.

\section{Conclusions}

In these interviews, analysts described the shortcomings of various technologies that they use, or technology features that they wish were available. These can be grouped into the following feature categories:

Data: Analysts want rapid access to all relevant data sources, advisories for data that may be relevant to their interests, and availability of information at the appropriate level for their analysis (e.g., output of interpretations from expert analysts instead of raw data).

Enhanced search: Analysts would like customization of information based on relevance, selective filtering of sources, prioritization of search topics, and the ability to view other analysts searches.

Verification: Analysts want indications of information that has been verified or discarded by other analysts, a trail of information history and uses, and automatic verification (e.g., data quality editing) if possible

Analytics: Analysts want access to forecasting models, services to suggest analysis methods, pointers to other analysts' expertise, methods, and reports, and tools for "big data" exploitation.

Collaboration and communication: Analysts want assistance identifying people who may have needed information, real-time chat, the ability to compare analyses with colleagues, and the ability to shield data, results, or collaborations from selected others.

Archival: Analysts want automation to provide lessons learned, methods and outcomes for related events, the ability to automatically improve baselines with analyzed data, and assistance with reporting on interim analytic decisions.

The current understanding of the biosurveillance analyst's functions and processes, based on the results of these interviews, will continue to evolve as further dialog with analysts are combined with results of evaluations during subsequent phases of the new BSVE program.

\section{Keywords}

biosurveillance; workflow; collaboration; operations

\section{Acknowledgments}

This work is sponsored by the Defense Threat Reduction Agency under Air Force Contract \#FA8721-05-C-0002. Opinions, interpretations, recommendations and conclusions are those of the authors and are not necessarily endorsed by the United States Government.

\author{
*Timothy Dasey \\ E-mail:TimD@LL.mit.edu
}

